\title{
Elongation of the styloid processes in kidney- transplanted patients: The role of ectopic calcification as possible cause of Eagle syndrome
}

\author{
Fulvia Costantinides, Federico Della Flora, Ingrid Tonni, Christiane Bodin, \\ Gabriele Bazzocchi, Mary Louise Artero, Gaetano Castronovo, Erica Vettori, \\ Vanessa Nicolin \& Roberto Di Lenarda
}

To cite this article: Fulvia Costantinides, Federico Della Flora, Ingrid Tonni, Christiane Bodin, Gabriele Bazzocchi, Mary Louise Artero, Gaetano Castronovo, Erica Vettori, Vanessa Nicolin \& Roberto Di Lenarda (2019): Elongation of the styloid processes in kidney-transplanted patients: The role of ectopic calcification as possible cause of Eagle syndrome, CRANIO®, DOI: 10.1080/08869634.2019.1640919

To link to this article: https://doi.org/10.1080/08869634.2019.1640919

曲 Published online: 11 Jul 2019.

Submit your article to this journal \lceil

山 Article views: 34

Q View related articles $\widetilde{ }$

View Crossmark data $\nearrow$ 


\title{
Elongation of the styloid processes in kidney-transplanted patients: The role of ectopic calcification as possible cause of Eagle syndrome
}

\author{
Fulvia Costantinides DDS, MSc ${ }^{\text {a }}$, Federico Della Flora DDS ${ }^{\mathrm{a}}$, Ingrid Tonni DDS, MSc, PhD ${ }^{\mathrm{b}}$, Christiane Bodin DDS, PhD', \\ Gabriele Bazzocchi MD, MSc, PhD ${ }^{d}$, Mary Louise Artero MD, MSce , Gaetano Castronovo MD, DDS, MSc ${ }^{\mathrm{a}}$, \\ Erica Vettori DDS, MSc ${ }^{a}$, Vanessa Nicolin PhD ${ }^{\mathrm{a}}$ and Roberto Di Lenarda DDS, MSc ${ }^{\mathrm{a}}$
}

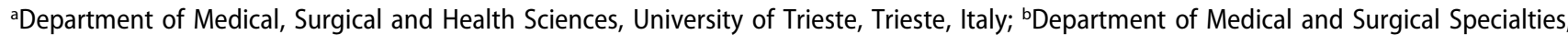
Radiological Sciences, and Public Health, University of Brescia, Brescia, Italy; 'Private practitioner, Brescia, Italy; ${ }^{\mathrm{d} D i v i s i o n}$ of Radiology, "Maggiore" University Hospital of Trieste, Trieste, Italy; eDivision of Nephrology and Dialysis, "Maggiore" University Hospital of Trieste, Trieste, Italy
\end{abstract}

ABSTRACT

Objective: To investigate the relationship between the presence of ectopic calcification in the elongation of the styloid process (SP) and its possible clinical manifestation (Eagle syndrome) in a population of kidney-transplant patients previously treated with hemodialysis.

Methods: Digital orthopantomography of 92 kidney-transplanted patients and 68 control subjects were analyzed to measure the length of SPs. Calcium, phosphate, alkaline phosphatase, and parathyroid hormone (PTH) blood levels were also available for comparison.

Results: The mean length of SPs was significantly different between groups, as were phosphate and PTH values.

Discussion: Renal transplant patients who have been treated with hemodialysis present elongated SPs, presumably due to alterations in phosphate balance and PTH levels. Thus, in cases of orofacial pain in patients with a history of altered bone metabolism, particularly when due to renal insufficiency, Eagle syndrome should be suspected as the main cause of symptoms.

\section{KEYWORDS}

Styloid process; Eagle

syndrome; kidney

transplantation;

orthopantomography;

hemodialysis; bone

metabolism; orofacial pain

\section{Introduction}

Eagle syndrome is an uncommon clinical manifestation of an elongation of the styloid process (SP) characterized by pain in the anterolateral region of the neck often with referred pain to the ear and sensation of a foreign body inside the pharynx region that prompts frequent swallowing, dysphagia, dysphonia, and persistent sore throat $[1,2]$.

An elongated SP can be found in $4 \%$ to $7.3 \%$ of the population, representing a rare condition that, in most cases, appears bilaterally $[3,4]$. When the calcified stylohyoid ligament is included, the prevalence increases to $28 \%, 33 \%$ or $78 \%$ [4-6]. The elongated SPs that cause symptoms are even less common; it has been estimated that approximately $4 \%$ to $10.3 \%$ of patients with an elongated SP present pain [7]. Although SP elongation appears bilaterally in most cases, bilateral symptoms are uncommon, and the patient generally presents with unilateral orofacial pain [8]. Eagle syndrome occurs mostly in women, and the anatomic variant of the stylohyoid complex is usually found in the elderly female population [9]. This predisposition is greater in patients between 60 and 79 years of age.

Although the mean length of the SP in the population ranges from 1.52 to $4.77 \mathrm{~cm}$ [10], Keur et al. suggested that if the ossified portion that appears on radiograms is longer than $30 \mathrm{~mm}$, it could be considered an elongated process [11]. As reported by Thot et al. [12], while length of SP is indeed a risk factor, positional variations in orientation (both anteriorly and medially) of the SP are also critical in Eagle syndrome presentation.

Diverse etiologic theories and pathogenic mechanisms associated with this condition are found in the literature [7,13-15]. Among these, the ossification of the stylohyoid complex has been associated with various systemic conditions characterized by altered calcium and phosphorus metabolism, such as end-stage renal disease (ESRD) [16].

The aim of this retrospective case-control study was to investigate the possible role of ectopic calcification in the elongation of the SP in a population of kidney transplant patients previously treated with 
hemodialysis for ESRD. The length of right and left SPs and serum values of calcium $(\mathrm{Ca})$, phosphate $(\mathrm{P})$, alkaline phosphatase $(\mathrm{AP})$, and parathormone $(\mathrm{PTH})$ were measured and compared with those of a control population.

\section{Materials and methods}

The study was carried out from March to September, 2016. The research was conducted in agreement with the guidelines of the Helsinki Declaration, as revised in 1975, and approved by the local Ethics Committee (Protocol n. 40/2010). Informed written consent was obtained from each patient.

Transplanted patients $(n=169)$ followed at the Department of Periodontology since the pretransplant hemodialysis period and 100 control patients consecutively treated at the Dental Emergency Department were initially recruited for the study. Transplanted patients presenting comorbidities associated with possible dystrophic calcification of the SP, such as previous trauma, scleroderma, dermatomyositis, systemic lupus erythematous, burns, neurological conditions, and myositis ossificans were excluded. In the control group, patients affected by renal insufficiency, alterations of bone metabolism, alteration in calcium and phosphate balance, or with conditions associated with ectopic calcifications of soft and hard tissues were excluded (data were collected both by medical history and by the patient's hospital dossier). In both groups, the subjects with orthopantomographies (OPT) that did not clearly show the SPs because of distortion or superimposition of other anatomic structures were excluded. Other clinical variables included age and sex of the subjects and mean duration of dialysis for treated patients.

Thus, the digital OPTs of 92 kidney transplant patients (G1) and 68 control patients (G2) were analyzed to measure the length of the right and left SPs.

Serum values of $\mathrm{Ca}, \mathrm{P}, \mathrm{AP}$, and PTH of G1 patients were collected from the medical records archived at the Division of Nephrology. Routine blood chemistries were obtained every six weeks and every six months for PTH in ESRD patients undergoing hemodialysis to monitor bone metabolism homeostasis. In this study, the last available values before kidney transplant were chosen, as this time corresponds to the maximal period on hemodialysis.

Given the retrospective design of the study, the values of Ca, P, AP, and PTH for G2 were not available and were extrapolated, assigning to each patient the higher value of the normal range for age and sex available from the hospital laboratory (Ca $8.4-10.2 \mathrm{mg} / \mathrm{dl} ;$ P $2.4-4.1 \mathrm{mg} / \mathrm{dl}$; AP: 45-115 U/l; PTH: $10-65$ pg/ml).

\section{Radiographic measurement of the styloid processes}

All the OPTs were performed using a digital device, Kodak 8000 (Kodak ${ }^{\oplus}$, Rochester, NY, USA), 140 kHz, $60-90 \mathrm{kV}, 2-15 \mathrm{~mA}$, an exposition time of $13.9 \mathrm{~s}$, and an image magnification factor of 1.27 . The patient was placed in an orthostatic position, with the Frankfurt plane parallel to the floor.

Measurement of the SPs on OPT images were carried out by the same operator (F.D.F.) with a dedicated function of a dental management software (GSO for Windows 7, Micromatica ${ }^{\circ}$, Verona, Italy) used for medical records and radiographic dental images. The operator was blinded regarding the groups (he was not aware if the OPT belonged to a G1 or G2 patient).

The anatomic limits of the SP were considered its apex (caudally) and the base of the internal acoustic meatus (cranially) [17]. Two different techniques were used for measuring the SP bilaterally. The first measured the length tracing a straight line from the apex to the base of the SP (T1) (Figure 1), and the second measured the SP tracing a curved line following its anatomic profile from the apex to the base (T2) (Figure 2). The decision to use two methods was

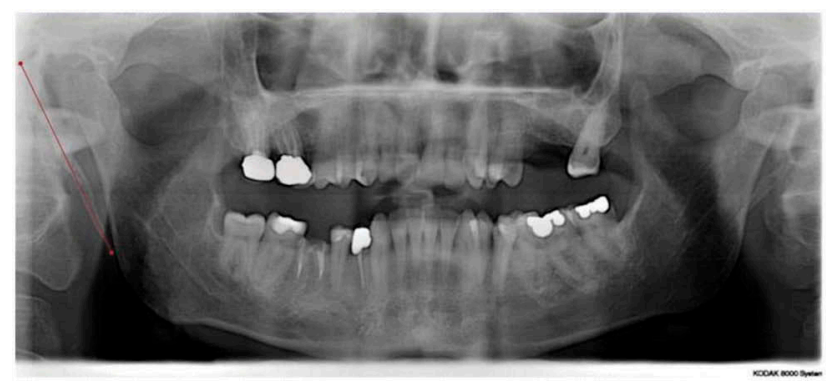

Figure 1. Right styloid process measured tracing a straight line from the apex to its base (T1).

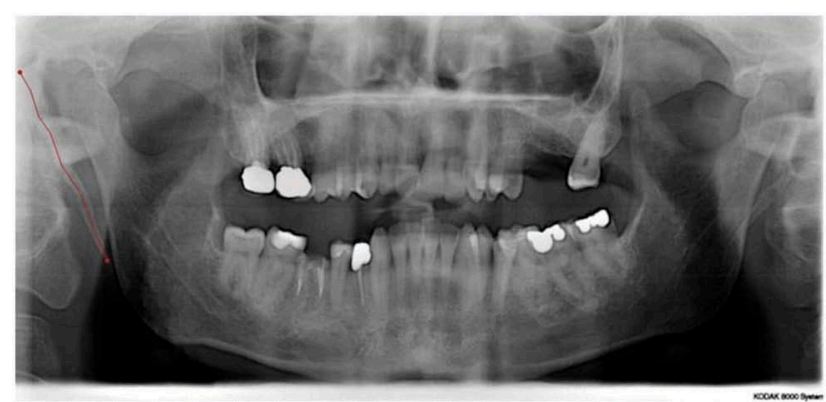

Figure 2. Right styloid process of the same patient measured tracing a curved line following its anatomic profile from the apex to the base (T2). 
based on testing the overlap of $\mathrm{T} 1$ and $\mathrm{T} 2$ in the measurement of the SP on OPT and eventually intercepting any statistically significant difference in the length of SP measured using a straight or curved line. The measurements $(\mathrm{x})$ were then converted considering the image magnification $(\mathrm{x} / 1.27)$. SPs longer than $30 \mathrm{~mm}$ were considered as elongated [11].

\section{Statistical analysis}

Data was processed using the SPSS software version 15.0 for Mac OS X (SPSS ${ }^{\circledR}$ Inc., Chicago, IL, USA).

Parametric methods were chosen after having tested the normality of the data using a Shapiro-Wilk test and the equality of variance among the datasets using Levene's test. The differences between groups in age and gender distribution were tested using the $t$-test for independent samples and the Fisher test, respectively. The difference in length of right and left SPs between T1 and T2 inside each group was analyzed using the $t$-test for paired data. The difference of the length of right and left SPs for T1 and T2 between groups was tested with $t$-test for independent samples. The difference of the mean values of $\mathrm{Ca}, \mathrm{P}, \mathrm{AP}$, and PTH between groups was tested with $t$-test for independent samples. The value of $p<.05$ was chosen as the level of statistical significance (two tailed).

\section{Results}

G1 consisted of 92 patients, 25 females and 67 males, with an age range of $30-85$ years (mean $59.69 \pm 13.65$ ) and mean dialysis duration time of $36.78 \pm 27.86$ months. G2 consisted of 68 patients, 26 females and 42 males with an age range of $38-87$ years (mean $60.18 \pm 13.36$ ). No statistical differences were found for gender distribution $(p=.17$; Fisher test) and mean age $(p=.82$; $t$-test). Differences between right and left SP measurements using T1 and T2 inside each group are shown in Table 1. The comparison between groups regarding the length of the right and left

Table 1. Comparison of mean right and left SP lengths measured using T1 and T2 inside each group. The differences are not significant ( $t$-test for paired data).

\begin{tabular}{llll}
\hline Group & T1 $(\mathrm{mm})(\mathrm{SD})$ & $\mathrm{T} 2(\mathrm{~mm})(\mathrm{SD})$ & $p$ \\
\hline G1 $(\mathrm{n}=92)$ & & & \\
Right SP & $39.12(10.20)$ & $40.96(10.59)$ & 0.10 \\
Left SP & $40.15(9.32)$ & $41.89(10.56)$ & 0.22 \\
G2 $(\mathrm{n}=68)$ & & & \\
Right SP & $21.71(8.11)$ & $21.46(8.19)$ & 0.51 \\
Left SP & $21.23(7.88)$ & $21.41(7.93)$ & 0.90 \\
\hline
\end{tabular}

SP: Styloid process; T1: Tracing 1; T2: Tracing 2; G1: Kidney transplant patients; G2: Control patients; SD: Standard deviation.
Table 2. Descriptive statistics and comparison of mean SP lengths and mean serum values of $\mathrm{Ca}, \mathrm{P}, \mathrm{AP}$, and PTH between groups ( $t$-test for independent samples).

\begin{tabular}{lccc}
\hline & $\mathrm{G} 1(\mathrm{n}=92)$ & $\mathrm{G} 2(\mathrm{n}=68)$ & $p$ \\
\hline Age (years) (SD) & $59.69(13.65)$ & $60.18(13.36)$ & 0.82 \\
Gender (M/F) & $67 / 25$ & $42 / 26$ & $0.17^{*}$ \\
Hemodialysis (months) (SD) & $36.78(27.86)$ & - & - \\
T1 - right SP (mm) (SD) & $39.12(10.20)$ & $21.71(8.11)$ & $<0.001$ \\
T1 - left SP (mm) (SD) & $40.15(9.32)$ & $21.23(7.88)$ & $<0.001$ \\
T2 - right SP (mm) (SD) & $40.96(10.59)$ & $21.46(8.19)$ & $<0.001$ \\
T2 - left SP (mm) (SD) & $41.89(10.56)$ & $21.41(7.93)$ & $<0.001$ \\
Ca (mg/dl) (SD) & $10.16(0.88)$ & $10.2(0.0)$ & 0.24 \\
P (mg/dl) (SD) & $5.42(1.83)$ & $4.1(0.0)$ & $<0.001$ \\
AP (pg/ml) (SD) & $96.69(62.87)$ & $115(0.0)$ & 0.06 \\
PTH (U/l) (SD) & $268.11(307.54)$ & $65(0.0)$ & $<0.001$ \\
\hline
\end{tabular}

*Fisher test; SP: Styloid process; Ca: Calcium; P: Phosphate; AP: Alkaline phosphatase; PTH: Parathyroid hormon; T1: Tracing 1; T2: Tracing 2; G1: Kidney transplant patients; G2: Control patients; SD: Standard deviation.

SPs measured with $\mathrm{T} 1$ and $\mathrm{T} 2$ and of $\mathrm{Ca}, \mathrm{P}, \mathrm{AP}$, and PTH values are described in Table 2.

\section{Discussion}

In the present research, a large population of kidney transplant patients (G1) previously treated with hemodialysis were studied to measure the length of SPs and to detect alterations in $\mathrm{Ca}$ and $\mathrm{P}$, which might be associated with an elongation of SPs. In addition to $\mathrm{Ca}$ and $\mathrm{P}, \mathrm{AP}$ and $\mathrm{PTH}$ were also analyzed. AP is an enzyme active in an alkaline environment at $\mathrm{pH} 8-10$ that is able to hydrolyze phosphate groups bound to inorganic substrates. AP promotes the primary mineralization and the bone remodeling. PTH is primarily involved in bone metabolism according to its concentration; at low levels, it leads to an increase of the bone mass, while at high levels, it stimulates osteoclastic activity with $\mathrm{Ca}$ mobilization from tissues and a consequent increase in calcemia. Results of SPs measurements and serum values were compared with those of a control population (G2).

Two techniques were used to measure the length of the SP on OPTs: T1 measured the length of SPs tracing straight lines, and T2 measured the SPs tracing a curved line. No differences were observed within each group regarding the technique used (Table 1). The results suggested that both T1 and T2 are useful methods for correctly measuring the length of SP. When comparing the mean length of the right and left SPs between groups for $\mathrm{T} 1$ and $\mathrm{T} 2$, a significant difference was found for each comparison. As shown in Table 2, right and left SPs of G1 were about two times longer than SPs of G2. This finding supports the hypothesis that a mechanism of ectopic calcification developed in patients with a persistent alteration of bone metabolism. The comparison of serum values showed a statistically significant difference for 
$p(p<.001 ; t$-test $)$ and PTH $(p<.001 ; t$-test $)$, while values of $\mathrm{Ca}$ and AP did not present differences between groups. However, it should be considered that serum concentration of the substances analyzed in G2 was extrapolated from the normal range; to avoid an overestimation of the differences with G1, the maximal possible values were used. According to this observation, the measurement of real values in the healthy population could bring out a significant difference also for $\mathrm{Ca}$ and AP.

Murtagh et al. have provided a plausible explanation regarding the etiologic theories associated with the elongation of SPs and the manifestation of Eagle syndrome [7]. The first is the theory of congenital elongation of the SP due to the persistence of the cartilaginous precursor; the second considers the calcification of the stylohyoid ligament by an unknown process; and the third suggests the growth of osseous tissue at the insertion of the stylohyoid ligament. Steinmann [15] proposed another list of theories to explain the ossification: 1) the theory of "reactive hyperplasia" when trauma activates the remnants of the original connective and fibrocartilaginous cells; 2) the theory of "reactive metaplasia," abnormal healing following trauma that initiates the ossification of the stylohyoid ligament; and 3) the theory of "anatomic variance" without any recognizable trauma. Camarda et al. [13] proposed a fourth theory in the absence of evident radiographic ossification: the theory of "aging developmental anomaly." In a recent review, Piagkou et al. [14] also posed a genetic "dysendocrine theory" and the correlation with rheumatoid disease.

Furthermore, the ossification of the stylohyoid complex has also been associated with various systemic conditions characterized by altered calcium, phosphorus, and vitamin D metabolism, such as ESRD [16]. Disturbances in the Ca-P balance cause prolonged stimulation of parathyroid glands with increased synthesis and release of PTH and secondary hyperparathyroidism (SHP). This last condition causes skeletal abnormalities known as renal osteodystrophy, which is associated with vascular and other soft tissue calcifications [18]. Only a single case report found in the current literature investigates the role of $\mathrm{Ca}$ and $\mathrm{P}$ metabolism in the elongation of the SPs [19]. The authors of this report concluded that ectopic calcification or ossification due to the abnormality in $\mathrm{Ca}$ and $\mathrm{P}$ metabolism might also have a role in the elongation of SP. However, this hypothesis is based only on a single patient.
As far as the authors know, the present study is the first to analyze the role of ectopic calcification in the development of elongated SPs in a large population using the alterations of bone metabolism in ESRD as a model.

Results highlight a strong association between altered bone metabolism and elongation of SPs explaining a possible pathogenic mechanism that could lead to the development of Eagle syndrome. However, results have to be used with caution due to the limitations of the study. The research is retrospective and was based only on the radiographic and laboratory findings, without considering the manifestation of clinical symptoms of Eagle syndrome, which is rare, in spite of the prevalence of SPs. Furthermore, the study analyzed the ossified stylohyoid complex without differentiating elongated SPs from calcified stylohyoid ligaments. Histological evaluations could clarify this point. Finally, no correlation has been made with the duration of the dialysis period, gender or age, factors that can influence the extent of ossification of SPs. Further studies are encouraged in this regard.

\section{Conclusion}

Within the limits of this study, it can be concluded that:

- ESRD represents a good model for the study of ectopic calcification in patients with systemic alterations of bone metabolism, as suggested by Sysman Y et al. [18];

- patients undergoing hemodialysis and with alterations of P and PTH present bilaterally elongated SPs;

- in case of orofacial pain in patients with proven alteration of bone metabolism, elongation of the styloid processes (Eagle syndrome) should be suspected and considered as the main cause of symptoms during the differential diagnosis.

\section{Disclosure statement}

No potential conflict of interest was reported by the authors.

\section{Funding}

No funding supported the work. 


\section{References}

[1] Costantinides F, Vidoni GBodin C, et al. Eagle's syndrome: signs and symptoms. Cranio. 2013;31(1):56-60.

[2] Kim E, Hansen K, Frizzi J. Eagle syndrome: case report and review of the literature. Ear Nose Throat J. 2008;87:631-633.

[3] Eagle WW. Elongated styloid process; symptoms and treatment. AMA Arch Otolaryngol. 1958;67:172-176.

[4] Kaufman SM, Elzay RP, Irish EF. Styloid process variation. Radiologic and clinical study. Arch Otolaryngol. 1970;91:460-463.

[5] Carroll MKO. Calcification in the stylohyoid ligament. Oral Surg Oral Med Oral Pathol. 1984;58:617-621.

[6] Miller DB. Eagle's syndrome and the trauma patient. Significance of an elongated styloid process and/or ossified stylohyoid ligament. Funct Orthod. 1997;14:30-35.

[7] Murtagh RD, Caracciolo JT, Fernandez G. CT findings associated with eagle syndrome. Am J Neuroradiol. 2001;22:1401-1402.

[8] Jan A. Stylohyoid syndrome. J Pak Med Assoc. 1989;39:23.

[9] Woolery WA. The diagnostic challenge of styloid elongation (Eagle's syndrome). J Am Osteopath Assoc. 1990;90:88-89.

[10] Mortellaro C, Biancucci P, Picciolo G, et al. Eagle's syndrome: importance of a corrected diagnosis and adequate surgical treatment. J Craniofac Surg. 2002;13:755-758.
[11] Keur JJ, Campbell JP, McCarthy JF, et al. The clinical significance of the elongated styloid process. Oral Surg Oral Med Oral Pathol. 1986 Apr;61(4):399-404.

[12] Thot B, Revel S, Mohandas R, et al. Eagle's syndrome. Anatomy of the styloid process. Indian J Dent Res. 2000;11:65-70.

[13] Camarda AJ, Deschamps C, Forest DI. Stylohyoid chain ossification: a discussion of etiology. Oral Surg Oral Med Oral Pathol. 1989;67:508-514.

[14] Piagkou M, Anagnostopoulou S, Kouladouros K, et al. Eagle's syndrome: a review of the literature. Clin Anat. 2009;22:545-558. Review.

[15] Steinmann EP. Styloid syndrome in absence of an elongated process. Acta Otolaryngol. 1968;66:347-356.

[16] Gokce C, Sisman Y, Ertas ET, et al. Prevalence of styloid process elongation on panoramic radiography in the Turkey population from cappadocia region. Eur J Dent. 2008;2:18-22.

[17] Natsis K, Repousi E, Noussios G, et al. The styloid process in a Greek population: an anatomical study with clinical implications. Anat Sci Int. 2015;90:67-74.

[18] Sisman Y, Gokce C, Sipahioglu M. Bilateral elongated styloid process in an end-stage renal disease patient with peritoneal dialysis: is there any role for ectopic calcification? Eur J Dent. 2009;3:155-157.

[19] Gokce C, Sisman Y, Sipahioglu M. Styloid process elongation or eagle's syndrome: is there any role for ectopic calcification? Eur J Dent. 2008;2:224-228. 